\title{
Quasi-Bound ${ }^{3} \mathrm{He}-\eta$ State and Spin-Observables of the Reaction $p d \rightarrow{ }^{3} \mathrm{He} \eta$
}

\author{
Yu.N. Uzikov ${ }^{1, a}$
}

Joint Institute for Nuclear Research, Moscow reg. Dubna, Joliot Curie, 6, 141980 Russia

\begin{abstract}
A formalism for total set of spin observables of the reaction $p d \rightarrow{ }^{3} \mathrm{He} \eta$ derived in a model independent way is presented and a possibility to perform a complete polarization experiment is investigated. Separation of s- and p-wave amplitudes of this reaction in the threshold region allows one to determine a position of the quasibound pole in the ${ }^{3} \mathrm{He}-\eta$ system. It is shown that in order to get such a separation one should measure spin-transfer coefficients in addition to analyzing powers and spin-correlations. Specific observables, given by products of one s- and one p-wave amplitudes, are found. Measurement of these observables will provide new independent information on the ${ }^{3} \mathrm{He}-\eta$ pole position and this allows one to determine whether the ${ }^{3} \mathrm{He}-\eta$ state is quasi-bound or anti-bound.
\end{abstract}

\section{Introduction}

Strong enhancement of the cross section of the reaction $p d \rightarrow{ }^{3} \mathrm{He} \eta$ observed near the threshold $[1,2]$ is considered as a final state interaction effect arising due to presence of a quasi-bound ${ }^{3} \mathrm{He}-\eta$ state [3]. This asumption is supported by the calculation of the cross section of this reaction within a two-step model [4-6]. Parameters of the quasi-bound state were estimated $[4,5]$ from fit to the data. Microscopic four-body calculations support quasi-bound [7] or anti-bound (virtual) [8] states in the ${ }^{3} \mathrm{He}-\eta$ system within the existing uncertainties for the elementary $\eta N$ interaction. A special type of experiments is required to decide whether the pole in the ${ }^{3} \mathrm{He}-\eta$ system, if it really exists, corresponds to a quasi-bound (for strong enough $\eta N$ interaction) or anti-bound (for rather weak $\eta N$ interaction) state [9].

Recently the cross section of the $p d \rightarrow{ }^{3} \mathrm{He} \eta$ reaction near its threshold was measured at COSY $[10,11]$ with a high precision. According to Ref.[10], the pole corresponding to the quasi-bound (or anti-bound) state is located on the excitation energy plane at the point $Q_{0}=$ $\left[\left(-0.30 \pm 0.15_{\text {stat }} \pm 0.04_{\text {syst }}\right) \pm l\left(0.21 \pm 0.29_{\text {stat }} \pm 0.06_{\text {syst }}\right] \mathrm{MeV}\right.$ that is very close to the threshold. As was noted in [12], the presence of the pole in the s-wave amplitude of this reaction must lead to fast variation not only in its magnitude, but also in the phase. The latter provides a new criterion for identification of the pole. The specific phase behaviour comes from the following analytical form of the s-wave amplitude near the pole:

$$
f\left(p_{\eta}\right)=\frac{\xi}{p_{\eta}-i p_{0}}
$$

\footnotetext{
${ }^{a}$ e-mail: uzikov@nusun.jinr.ru
}

where $p_{\eta}$ is the (real) c.m.s. momentum of the $\eta$-meson, $p_{0}$ is the (complex) pole point directly related to the energy $Q_{0}$, and $\xi$ is a smooth function of $p_{\eta}$. On the other hand, p-wave (and other higher partial waves) amplitudes are expected to exhibit non-pole behaviour. Using the available unpolarized data [10], the authors of Ref.[12] found some non-direct indications of this specific phase behaviour of the s-wave amplitudes near the threshold. In order to validate this interpretation by direct measurement of energy dependence of the s-wave amplitudes, polarization experiments are required. Some of them were discussed in Ref. [12] under restriction by four spin amplitudes in the transition matrix element.

In paper [13] polarization measurements were discussed in detail. A full set of spin observables was analyzed including those which require measurement of polarizations of final particles. Moreover, in addition to the approximation by four spin amplitudes, used in Ref.[12], the case with five amplitudes and the general case, which includes a full set of six independent spin amplitudes of this reaction were considered. It was shown that in collinear kinematics the complete polarization experiment is rather simple, but one cannot separate s- and p- wave amplitudes in this regime. Therefore, to determine the desirable phase dependence of the s-wave amplitudes in respect of $p$-wave ones one has to perform experiments beyond collinear kinematics. For the cases of four and five independent amplitudes complete polarization experiments were suggested, which allow one to determine all these amplitudes. It was found that one has to measure spin-transfer coefficients in addition to analyzing powers and spin-correlation parameters to complete the experiment. Furthermore, it was shown that in the most general case with six spin amplitudes the complete polarization experiment is too complicated to be really performed. Instead of performing it, it was suggested 
in Ref. [13] to measure few specific spin observables which could provide new independent information on the position of the quasi-bound pole. In this talk I will focus mainly on these specific spin observables.

\section{The transition matrix element}

In terms of z-projections of spins of participating particles there exist twelve different spin transitions in the reaction $p d \rightarrow{ }^{3} \mathrm{He} \eta$. Due to P-parity and angular momentum conservation only six of them are independent [13].

In the non-orthogonal basis used in Ref.[12] and in notations of that paper, the transition operator of the reaction $p d \rightarrow{ }^{3} \mathrm{He} \eta$ can be written as

$$
\begin{aligned}
& \hat{F}=\boldsymbol{\varepsilon} \cdot \mathbf{T}=A \boldsymbol{\varepsilon} \cdot \hat{\mathbf{p}}_{p}+i B[\boldsymbol{\varepsilon} \times \boldsymbol{\sigma}] \cdot \hat{\mathbf{p}}_{p}+C \boldsymbol{\varepsilon} \cdot \mathbf{p}_{\eta}+ \\
& i D[\boldsymbol{\varepsilon} \times \boldsymbol{\sigma}] \cdot \mathbf{p}_{\eta}+i E(\boldsymbol{\varepsilon} \cdot \mathbf{n})\left(\boldsymbol{\sigma} \cdot \hat{\mathbf{p}}_{p}\right)+i F(\boldsymbol{\varepsilon} \cdot \mathbf{n})\left(\boldsymbol{\sigma} \cdot \mathbf{p}_{\eta}\right),(2)
\end{aligned}
$$

where $\boldsymbol{\sigma}$ is the Pauli matrix, $\boldsymbol{\varepsilon}$ is the polarization vector of the deuteron, $\hat{\mathbf{p}}_{p}$ is the unit vector along the proton beam direction, $\mathbf{p}_{p}$ and $\mathbf{p}_{\eta}$ are the cms momenta of the proton and the $\eta$-meson, respectively, and $\mathbf{n}=\left[\mathbf{p}_{\eta} \times \hat{\mathbf{p}}_{p}\right]$. We choose the coordinate system with the axes OZ $\uparrow \uparrow \mathbf{p}_{p}$, OY $\uparrow \uparrow \mathbf{n}$, OX $\uparrow \uparrow\left[\mathbf{n} \times \hat{\mathbf{p}}_{p}\right]$. Six independent terms in Eq.(2) completely describe this reaction. As compared to Ref.[12], we also consider two additional terms, $\mathrm{E}$ and $\mathrm{F}$, both of the non-s-wave type (in respect of the final state). The swave amplitudes are contained in the terms $A$ and $B$ only. These are the only terms which do not disappear in the limit $p_{\eta} \rightarrow 0$ in Eq. (2). In the near threshold region, the terms $A$ and $B$ could contain mainly an admixture of pwaves, like $A^{\prime}\left(\mathbf{p}_{p} \cdot \mathbf{p}_{\eta}\right)\left(\boldsymbol{\varepsilon} \cdot \mathbf{p}_{p}\right)$ and $i B^{\prime}\left(\mathbf{p}_{p} \cdot \mathbf{p}_{\eta}\right)[\boldsymbol{\varepsilon} \times \boldsymbol{\sigma}] \cdot \hat{\mathbf{p}}_{p}$, respectively. The terms $C, D$ and $E$ are related to the final p-wave state near the threshold. The last term $F$ in Eq. (2) corresponds to the d-wave (and higher partial waves) in the final state.

The unpolarized $\mathrm{cms}$ cross section takes the form (see also below Eqs. (8) and (9))

$$
d \sigma_{0}=\frac{p_{\eta}}{3 p_{p}} I
$$

here the factor $I$ is given as

$$
\begin{array}{r}
I=|A|^{2}+2|B|^{2}+\left(|C|^{2}+2|D|^{2}\right) p_{\eta}^{2}+ \\
+2 \operatorname{Re}\left(A C^{*}+2 B D^{*}\right) p_{\eta} \cos \theta_{\eta}+\left(|F|^{2} p_{\eta}^{2}+|E|^{2}\right) \times \\
\times p_{\eta}^{2} \sin ^{2} \theta_{\eta}+2 \operatorname{Re}\left(D E^{*}-B F^{*}+E F^{*} p_{\eta} \cos \theta_{\eta}\right) \times \\
\times p_{\eta}^{2} \sin ^{2} \theta_{\eta}
\end{array}
$$

with $\theta_{\eta}$ being the angle between the vectors $\mathbf{p}_{p}$ and $\mathbf{p}_{\eta}$. If one puts $F=0$ and $E=0$, Eq. (4) gives linear dependence in $\cos \theta_{\eta}$ for the differential cross section, observed in the existing data at $Q<11 \mathrm{MeV}[10,11]$. In view of this observation, one may conclude the following. Firstly, the contribution of two last terms in Eq.(4), which are proportional to $\sin ^{2} \theta_{\eta}$, can be considered as negligible in the unpolarized cross section at $Q$ less than $\approx 10 \mathrm{MeV}$. The latter gives grounds for using only four independent amplitudes and neglecting $E$ and $F$. This approximation, adopted in Ref.[12], was also studied in Ref. [13] from the point of view of performing a complete polarization experiment. Secondly, the amplitudes $A$ and $B$ do not contain a sizeable contribution of p-waves (i.e. terms $A^{\prime}$ and $B^{\prime}$ considered above) at $Q<10 \mathrm{MeV}$ [12]

Thus, near the threshold the amplitudes $A$ and $B$ are of the s-wave type and, therefore, are expected to contain the quasi-bound pole. On the other hand, all other amplitudes $(C, D, E$, and $F)$ are expected to have smooth $p_{\eta}$ dependence near the pole. These assumptions will be essential in section 5, where the position of the quasi-bound pole on the momentum plane is considered. One should note that the d-wave term $F$ is most likely negligible as compared to the s- and p-wave terms in the near threshold region due to the centrifugal barrier. However, the term $E$ could be nonnegligible in spin observables. Therefore, the cases with five spin amplitudes $(A, B, C, D, E)$ and the general case with the full set of six spin amplitudes have to also considered (see Ref. [13]).

\section{Observables}

Using Eq. (2), one can express any spin observables in terms of invariant spin amplitudes. On the next step, having the full set of spin observables, one can find a solution for complete polarization experiments. For this aim the Cartesian components of the vector-operator $\mathbf{T}$, defined by Eq. (2), can be presented as follows

$$
\begin{aligned}
& T_{x}=M_{1}+M_{2} \sigma_{y}, T_{y}=M_{3} \sigma_{x}+M_{4} \sigma_{z} \\
& T_{z}=M_{5}+M_{6} \sigma_{y}
\end{aligned}
$$

where

$$
\begin{aligned}
& M_{1}=C q_{x}, M_{2}=i\left(B+D q_{z}\right), \\
& M_{3}=-i\left(B+D q_{z}-F q_{x}^{2}\right), M_{4}=i\left(D+E+F q_{z}\right) q_{x}, \\
& M_{5}=A+C q_{z}, M_{6}=-i D q_{x} .
\end{aligned}
$$

Here $q_{x}$ and $q_{z}$ are determined as

$$
q_{x}=-p_{\eta} \sin \theta_{\eta}, q_{z}=p_{\eta} \cos \theta_{\eta} .
$$

The unpolarized cross section can be written as

$$
d \sigma_{0}=\frac{1}{6} \frac{p_{\eta}}{p_{p}} \sum_{\alpha} \operatorname{Tr} T_{\alpha} T_{\alpha}^{+}=\frac{1}{3} \frac{p_{\eta}}{p_{p}} I,
$$

where $\alpha=x, y, z$ and $I$ has the following form:

$$
I=\frac{1}{2} \sum_{\alpha} \operatorname{Tr} T_{\alpha} T_{\alpha}^{+}=\sum_{i=1}^{6}\left|M_{i}\right|^{2} .
$$

We use below definitions and notations of Ref. [14] for spin observables. The full set of observables for the general case of six independent spin amplitudes is given in Ref. [13]. Particular cases with five and four spin amplitudes, which are expected to domiante near the threshold of the reaction $p d \rightarrow{ }^{3} \mathrm{He} \eta$, are also considered in Ref.[13]. 
$19^{\text {th }}$ International IUPAP Conference on Few-Body Problems in Physics

Here we present mainly those observables which are required for determenitaion of the optimal (specific) spinobserbvabes. form

The tensor analyzing powers of the deuteron take the

$$
\begin{aligned}
A_{y y} & =1-\frac{3}{I}\left\{\left|M_{3}\right|^{2}+\left|M_{4}\right|^{2}\right\}, \\
A_{x x} & =1-\frac{3}{I}\left\{\left|M_{1}\right|^{2}+\left|M_{2}\right|^{2}\right\}, \\
A_{z z} & =1-\frac{3}{I}\left\{\left|M_{5}\right|^{2}+\left|M_{6}\right|^{2}\right\}, \\
-\frac{I}{3} A_{x z} & =\operatorname{Re}\left(M_{1} M_{5}^{*}+M_{2} M_{6}^{*}\right) .
\end{aligned}
$$

The vector analyzing powers are

$$
\begin{aligned}
& \frac{I}{2} A_{y}^{d}=\operatorname{Im}\left\{M_{5} M_{1}^{*}+M_{6} M_{2}^{*}\right\}, \\
& \frac{I}{2} A_{y}^{p}=\operatorname{Re}\left\{M_{1} M_{2}^{*}+M_{5} M_{6}^{*}\right\}-\operatorname{Im} M_{3} M_{4}^{*} .
\end{aligned}
$$

The spin transfer coefficients $K_{i}^{j^{\prime}}(p)$, describing polarization transfer from the initial proton to the ${ }^{3} \mathrm{He}$ nucleus, can be written as

$$
\begin{aligned}
I K_{y}^{y^{\prime}}(p) & =\left|M_{1}\right|^{2}+\left|M_{2}\right|^{2}-\left|M_{3}\right|^{2}-\left|M_{4}\right|^{2}+\left|M_{5}\right|^{2}+\left|M_{6}\right|^{2}, \\
I K_{x}^{x^{\prime}}(p) & =\left|M_{1}\right|^{2}-\left|M_{2}\right|^{2}+\left|M_{3}\right|^{2}-\left|M_{4}\right|^{2}+\left|M_{5}\right|^{2}-\left|M_{6}\right|^{2}, \\
I K_{z}^{z^{\prime}}(p) & =\left|M_{1}\right|^{2}-\left|M_{2}\right|^{2}-\left|M_{3}\right|^{2}+\left|M_{4}\right|^{2}+\left|M_{5}\right|^{2}-\left|M_{6}\right|^{2}, \\
-\frac{I}{2} K_{x}^{z^{\prime}}(p) & =\operatorname{Im}\left\{M_{1} M_{2}^{*}+M_{5} M_{6}^{*}\right\}-\operatorname{Re}_{3} M_{4}^{*}, \\
-\frac{I}{2} K_{z}^{x^{\prime}}(p) & =\operatorname{Im}\left\{M_{2} M_{1}^{*}+M_{6} M_{5}^{*}\right\}-\operatorname{Re}_{3} M_{4}^{*} .
\end{aligned}
$$

The spin transfer coefficients $K_{j}^{i^{\prime}}(d)$, describing polarization transfer from the initial deuteron to the ${ }^{3} \mathrm{He}$ nucleus, are

$$
\begin{gathered}
\frac{I}{2} K_{y}^{y^{\prime}}(d)=\operatorname{Im}\left(M_{6} M_{1}^{*}+M_{5} M_{2}^{*}\right), \\
\frac{I}{2} K_{x}^{x^{\prime}}(d)=\operatorname{Im} M_{3} M_{5}^{*}-\operatorname{Re} M_{4} M_{6}^{*}, \\
\frac{I}{2} K_{z}^{z^{\prime}}(d)=\operatorname{Im} M_{1} M_{4}^{*}-\operatorname{Re}_{2} M_{3}^{*}, \\
\frac{I}{2} K_{z}^{x^{\prime}}(d)=\operatorname{Im} M_{1} M_{3}^{*}+\operatorname{Re} M_{2} M_{4}^{*}, \\
\frac{I}{2} K_{x}^{z^{\prime}}(d)=\operatorname{Im} M_{4} M_{5}^{*}+\operatorname{Re}_{3} M_{6}^{*} .
\end{gathered}
$$

The proton-deuteron spin correlation parameters are

$$
\begin{aligned}
-\frac{I}{2} C_{y, y} & =\operatorname{Im} M_{2} M_{5}^{*}+\operatorname{Im} M_{1} M_{6}^{*}, \\
\frac{I}{2} C_{z, z} & =\operatorname{Im} M_{1} M_{4}^{*}+\operatorname{Re} M_{2} M_{3}^{*}, \\
\frac{I}{2} C_{x, x} & =\operatorname{Im} M_{3} M_{5}^{*}+\operatorname{Re}_{4} M_{6}^{*}, \\
\frac{I}{2} C_{x, z} & =\operatorname{Im} M_{1} M_{3}^{*}-\operatorname{Re}_{2} M_{4}^{*}, \\
\frac{I}{2} C_{z, x} & =\operatorname{Im}_{4} M_{5}^{*}-\operatorname{Re}_{3} M_{6}^{*}
\end{aligned}
$$

for the vector polarized deuteron and

$$
\begin{aligned}
C_{y, y y} & =A_{y}^{p}+\frac{6}{I} \operatorname{Im}_{3} M_{4}^{*}, \\
\frac{I}{2} C_{y, y y} & =\operatorname{Re}\left(M_{1} M_{2}^{*}+M_{5} M_{6}^{*}\right)+2 \operatorname{Im} M_{3} M_{4}^{*}, \\
C_{y, x x} & =A_{y}^{p}-\frac{6}{I} \operatorname{Re} M_{2} M_{1}^{*}, \\
\frac{I}{2} C_{y, x x} & =\operatorname{Re}\left(M_{5} M_{6}^{*}-2 M_{2} M_{1}^{*}\right)-\operatorname{Im} M_{3} M_{4}^{*}, \\
C_{y, z z} & =A_{y}^{p}-\frac{6}{I} \operatorname{Re} M_{5} M_{6}^{*}, \\
\frac{I}{2} C_{y, z z} & =\operatorname{Re}\left(M_{1} M_{2}^{*}-2 M_{5} M_{6}^{*}\right)-\operatorname{Im} M_{3} M_{4}^{*}, \\
-\frac{I}{3} C_{y, x z} & =\operatorname{Re}\left(M_{2} M_{5}^{*}+M_{1} M_{6}^{*}\right), \\
-\frac{I}{3} C_{x, y z} & =\operatorname{Re} M_{3} M_{5}^{*}-\operatorname{Im} M_{4} M_{6}^{*}, \\
-\frac{I}{3} C_{z, y z} & =\operatorname{Re} M_{4} M_{5}^{*}-\operatorname{Im} M_{6} M_{3}^{*}, \\
-\frac{I}{3} C_{x, x y} & =\operatorname{Re} M_{1} M_{3}^{*}-\operatorname{Im} M_{4} M_{2}^{*}, \\
-\frac{I}{3} C_{z, x y} & =\operatorname{Re} M_{1} M_{4}^{*}-\operatorname{Im} M_{2} M_{3}^{*}
\end{aligned}
$$

for the tensor polarized deuteron. Polarization transfer from the tensor polarized deuteron to the ${ }^{3} \mathrm{He}$ nucleus is described by the following observables:

$$
\begin{aligned}
K_{y y}^{y^{\prime}} & =P_{y}^{h}+\frac{6}{I} \operatorname{Im} M_{4} M_{3}^{*}, \\
\frac{I}{2} K_{y y}^{y^{\prime}} & =\operatorname{Re}\left(M_{1} M_{2}^{*}+M_{5} M_{6}^{*}\right)-2 \operatorname{Im} M_{4}^{*} M_{3}, \\
K_{x x}^{y^{\prime}} & =P_{y}^{h}-\frac{6}{I} \operatorname{Re} M_{2} M_{1}^{*}, \\
\frac{I}{2} K_{x x}^{y^{\prime}} & =\operatorname{Re}\left(M_{5} M_{6}^{*}-2 M_{1} M_{2}^{*}\right)+\operatorname{Im} M_{3} M_{4}^{*}, \\
K_{z z}^{y^{\prime}} & =P_{y}^{h}-\frac{6}{I} \operatorname{Re} M_{5} M_{6}^{*}, \\
\frac{I}{2} K_{z z}^{y^{\prime}} & =\operatorname{Re}\left(M_{1} M_{2}^{*}-2 M_{5} M_{6}^{*}\right)+\operatorname{Im} M_{3} M_{4}^{*}, \\
-\frac{I}{3} K_{y z}^{x^{\prime}} & =\operatorname{Re} M_{3} M_{5}^{*}+\operatorname{Im} M_{4} M_{6}^{*}, \\
-\frac{I}{3} K_{x y}^{z^{\prime}} & =\operatorname{Re} M_{1} M_{4}^{*}+\operatorname{Im} M_{2} M_{3}^{*}, \\
-\frac{I}{3} K_{x z}^{y^{\prime}} & =\operatorname{Re}\left(M_{2} M_{5}^{*}+M_{1} M_{6}^{*}\right), \\
-\frac{I}{3} K_{y z}^{z^{\prime}} & =\operatorname{Re} M_{4} M_{5}^{*}+\operatorname{Im} M_{6} M_{3}^{*}, \\
-\frac{I}{3} K_{x y}^{x^{\prime}} & =\operatorname{Re} M_{1} M_{3}^{*}+\operatorname{Im} M_{2}^{*} M_{4},
\end{aligned}
$$

where $P_{y}^{\tau}$ is the polarization of the final ${ }^{3} \mathrm{He}$ nucleus for the unpolarized beam and target, which can be written as

$$
\begin{aligned}
\frac{I}{2} P_{y}^{\tau} & =\operatorname{Re}\left(M_{1} M_{2}^{*}+M_{5} M_{6}^{*}\right)+\operatorname{Im} M_{3} M_{4}^{*}, \\
P_{x}^{\tau} & =P_{z}^{\tau}=0 .
\end{aligned}
$$




\section{Complete polarization experiment}

\subsection{Collinear kinematics}

In collinear kinematics $\left(q_{x}=0\right)$ one has $M_{1}=M_{4}=M_{6}=$ 0 and $M_{3}=-M_{2}$, as follows from Eq. (6). Therefore, only two spin amplitudes, $M_{2}=M_{2}^{\text {coll }}$ and $M_{5}=M_{5}^{\text {coll }}$, completely describe the reaction. These amplitudes (moduli and phases) can be determined from the measurement of the following four observables: $d \sigma_{0}, A_{z z}, C_{y, y}$ and $C_{y, x z}$ The relative phase $\phi_{i j}$ of the amplitudes $M_{i}$ and $M_{j}$ is defined as $\operatorname{Re} M_{i} M_{j}^{*}=\left|M_{i}\right|\left|M_{j}\right| \cos \phi_{i j}, \operatorname{Im} M_{i} M_{j}^{*}=\left|M_{i}\right|\left|M_{j}\right| \sin \phi_{i j}$. In the general case, $M_{2}^{\text {coll }}$ and $M_{5}^{\text {coll }}$ are the following combinations of the s- and p-wave amplitudes: $M_{5}^{\text {coll }}=A \pm C p_{\eta}$ and $M_{2}^{\text {coll }}=B \pm D p_{\eta}$, where the \pm sign refers to forward and backward production of the $\eta$ meson, respectively. Obviously, in collinear kinematics one cannot separate s- and p-wave amplitudes [13]. The only exception is the limiting point $p_{\eta}=0$. At this point the $\mathrm{p}$-wave amplitudes vanish and the separation of two s-wave amplitudes can be performed exactly.

\subsection{Four spin amplitudes}

If one puts $E=F=0$ in Eq.(2), then one has $M_{2}=-M_{3}$ and $M_{4}=-M_{6}$. In this case the spin structure of Eq. (2) coincides with that used in Ref. [12]. Thus, the following replacements in Eqs.(8)-(21)

$$
\begin{aligned}
& M_{1}=C q_{x}, M_{2}=i\left(B+D q_{z}\right), M_{3}=-M_{2}, \\
& M_{4}=i D q_{x}, M_{5}=A+C q_{z}, M_{6}=-M_{4}
\end{aligned}
$$

reduce the general case to the approximation by four spin amplitudes. The spin observables in terms of the amplitudes $A, B, C$ and $D$ for this case are given in the Appendix of Ref.[13].

When neglecting the terms $E$ and $F$ in Eq. (2), one can find the only relation which contains moduli of the amplitudes and does not involve their phases:

$$
\begin{aligned}
\frac{I}{2}\left(C_{y, y}-C_{x, x}+C_{z, z}\right)= & \frac{I}{3}\left(1-A_{y y}\right)= \\
& |D|^{2} q_{x}^{2}+\left|B+D q_{z}\right|^{2} .
\end{aligned}
$$

However, this relation does not provide new information as compared to $A_{y y}$. Furthermore, taking into account the relation

$$
A_{x x}+A_{y y}+A_{z z}=0,
$$

one can find [13] that the unpolarized cross section $d \sigma_{0}$, tensor analyzing powers $A_{i j}$ and spin correlation coefficients $C_{i, j}$ and $C_{i, j k}$ allow one to derive only three independent linear equations containing the squared moduli of the following four amplitudes: $M_{a}=A+C q_{z}, M_{b}=B+D q_{z}, C$ and $D$. Therefore, one cannot determine the moduli independently of the phases of these amplitudes and has to find all of them simultaneously. For this aim, one can use nine observables $d \sigma_{0}, A_{x x}, A_{y y}, A_{x z}, C_{y, y}, C_{x, x}, C_{z, z}, C_{x, z}$, and $C_{z, x}$, which give nine (nonlinear) equations connecting four moduli and six cosines of phases $\cos \phi_{C D}, \cos \phi_{a b}$, $\cos \phi_{C a}, \cos \phi_{C b}, \cos \phi_{D a}, \cos \phi_{D b}$. Due to Eq. (23), only eight equations are independent. Furthermore, since only three relative phases are independent, one should add to these system the following three linear equations connecting six relative phases: $\phi_{C b}-\phi_{C a}=\phi_{a b}, \phi_{D b}-\phi_{D a}=\phi_{a b}$, $\phi_{C b}-\phi_{D b}=\phi_{C D}$. However, in order to really use these relations one also has to employ equations containing sines of the phases. Therefore, one should take, for example, the following six observables $A_{y}^{d}, A_{y}^{p}, C_{y, y y}, C_{y, z z}, C_{x, y z}$, and $C_{z, x y}$ [13]. In total, one has 17 equations, which contain 14 spin observables and 16 unknown variables (four moduli, six cosines and six sines of the phases). Most likely, a solution to this system of equations can be found numerically, but this version of complete polarization experiment is too cumbersome.

The problem becomes much simpler if one can measure spin transfer coefficients. A possible solution is the following. The moduli of the amplitudes $M_{a}=A+C q_{z}$, $M_{b}=B+D q_{z}, D$ and $C$ can be found from the measurement of $d \sigma_{0}, A_{y y}, A_{x x}$ and $K_{x}^{x^{\prime}}(p)$ as

$$
\begin{aligned}
|C|^{2} q_{x}^{2} & =\frac{I}{3}\left(2 A_{y y}-A_{x x}-1\right)+\frac{I}{2}\left[1-K_{x}^{x^{\prime}}(p)\right], \\
\left|B+D q_{z}\right|^{2} & =\frac{2 I}{3}\left(1-A_{y y}\right)-\frac{I}{2}\left[1-K_{x}^{x^{\prime}}(p)\right], \\
|D|^{2} q_{x}^{2} & =-\frac{I}{3}\left(1-A_{y y}\right)+\frac{I}{2}\left[1-K_{x}^{x^{\prime}}(p)\right], \\
\left|A+C q_{z}\right|^{2} & =\frac{I}{3}\left(2+A_{x x}\right)-\frac{I}{2}\left[1-K_{x}^{x^{\prime}}(p)\right] .
\end{aligned}
$$

In order to completely determine four complex amplitudes three relative phases have to be measured (note that the common phase is non-measurable and one can put it equal to zero). A possible solution for the relative phases of the amplitudes $C, M_{b}, D$, and $M_{a}$, whose moduli are given in Eqs.(25), (26), (27) and (28) is to measure $K_{x}^{z^{\prime}}(p), K_{z}^{x^{\prime}}(p)$ and $K_{x}^{z^{\prime}}(d)$. It gives $\cos \phi_{D b}, \cos \phi_{C b}$ and $\cos \phi_{D a}$, as it follows from the following relations:

$$
\begin{aligned}
I\left[K_{x}^{z^{\prime}}(p)+K_{z}^{x^{\prime}}(p)\right]= & -2 \operatorname{Re}^{*}\left(B+D q_{z}\right) q_{x}, \\
I\left[K_{x}^{z^{\prime}}(p)-\frac{1}{2} K_{x}^{z^{\prime}}(d)\right]= & q_{x} \operatorname{Re}\left(B+D q_{z}\right) C^{*}, \\
I\left[K_{x}^{z^{\prime}}(p)-K_{z}^{x^{\prime}}(p)\right]= & -2 q_{x}\left[\operatorname{Re}\left(B+D q_{z}\right) C^{*}-\right. \\
& \left.\left(A+C q_{z}\right) D^{*}\right] .
\end{aligned}
$$

In order to find $\sin \phi_{D a}, \sin \phi_{D b}$ and $\sin \phi_{C b}$, one has to measure $C_{y, y y}, C_{y, x x}$ and $A_{y}^{p}$ [13]. Thus, in this version one has to perform ten accurate measurements.

\subsection{Five spin amplitudes}

Neglecting the d-wave amplitude $F$ in Eq. (2), one has the rerlation $M_{2}=-M_{3}$. For this case moduli of five amplitudes can be found by measurement of $d \sigma_{0}, A_{x x}, A_{y y}$, $K_{x}^{x^{\prime}}(p)$ and $K_{z}^{z^{\prime}}(p)$. In order to find their phases one need in addition 8 accurate measurements, for example, $K_{x}^{z^{\prime}}(d)$, $C_{z, x}, C_{z, y z}, K_{y z}^{z^{\prime}}, C_{x, z}, K_{z}^{x^{\prime}}(d), C_{x, x y} K_{x y}^{x^{\prime}}[13]$. 
$19^{\text {th }}$ International IUPAP Conference on Few-Body Problems in Physics

\subsection{Full set of spin amplitudes}

In the general case, when all six spin amplitudes $M_{i}(i=$ $1, \ldots, 6)$ are included in the consideration, there is no simple way to determine moduli of these amplitudes. Therefore, as was concluded in Ref. [13], the complet polarization experiment becomes too complicated and, most likely, unrealistic.

\section{Specific spin observables}

The complete polarization experiment provides full information about the reaction, including possible presence and position of the quasi-bound pole. However, in order to find solely the specific phase behaviour of the s-wave amplitudes caused by the ${ }^{3} \mathrm{He}-\eta$ pole, one should select only those observables which are most informative in this respect. In this connection one should note that at $q_{z}=0$ the amplitudes $M_{2}, M_{3}$ and $M_{5}$ are of the s-wave type and, therefore, are expected to contain the ${ }^{3} \mathrm{He}-\eta$ pole. On the other hand, the p- and d- wave amplitudes $M_{1}$, $M_{4}$ and $M_{6}$ are of the non-pole type with smooth $p_{\eta}$ dependence. As shown in Ref. [?], in some cases one can get a product of s-wave and $\mathrm{p}$ - wave amplitudes without performing a complete polarization experiment. Indeed, as follows from Eqs. (17), (18), (19) and (20), the observables $K_{z}^{x^{\prime}}(d), C_{x, z}, C_{x, x y}$ and $K_{x y}^{x^{\prime}}$ determine the following complex numbers $M_{1}^{*} M_{3}$ and $M_{4}^{*} M_{2}: \operatorname{Re}_{2} M_{4}^{*}=\frac{I}{4}\left[K_{z}^{x^{\prime}}(d)-\right.$ $\left.C_{x, z}\right], \operatorname{Im} M_{2} M_{4}^{*}=\frac{I}{6}\left[K_{x y}^{x^{\prime}}-C_{x, x y}\right]$, which can be written as

$$
\begin{aligned}
Z_{1}= & M_{2} M_{4}^{*}=q_{x}\left[B\left(D^{*}+E^{*}\right)+\left[D\left(E^{*}+D^{*}\right)+\right.\right. \\
& \left.\left.\left.(B+D) F^{*}\right)\right] q_{z}\right], \\
Z_{2}= & M_{3} M_{1}^{*}=-i q_{x}\left[B C^{*}+D C^{*} q_{z}-F C^{*} q_{x}^{2}\right] .
\end{aligned}
$$

At $q_{z}=0$, both of them are products of one s-wave and one p-wave amplitude, because the contribution of the d-wave term $F q_{x}^{2}$ to Eq. (31) is negligible in the near threshold region. Therefore, $Z_{1}$ and $Z_{2}$ are expected to have the pole form of Eq. (1).

Likewise, one can find from Eqs.(17), (18), (19) and (20) that four observables $K_{x}^{z^{\prime}}(d), C_{z, x}, C_{z, y z}$ and $K_{y z}^{z^{\prime}}$ completely determine the following complex numbers:

$$
\begin{aligned}
Z_{3}= & M_{5} M_{4}^{*}=-i q_{x}\left[A\left(D^{*}+E^{*}\right)+\right. \\
& \left.+\left(A F^{*}+C D^{*}+C E^{*}\right) q_{z}+C F^{*} q_{z}^{2}\right], \\
Z_{4}= & M_{3} M_{6}^{*}=\left[B D^{*}+|D|^{2} q_{z}-F D^{*} q_{x}^{2}\right] q_{x},
\end{aligned}
$$

which are also expected to have the same pole form at $q_{z} \rightarrow 0$. Thus, even without knowing the moduli of the amplitudes $\left|M_{i}\right|(i=1, \ldots, 6)$, one can single out the pole dependence (1) of the measured complex numbers $Z_{k}(k=$ $1, \ldots, 4)$.

Furthermore, using Eq. (1), where $f\left(p_{\eta}\right)=Z_{k}\left(p_{\eta}\right)$, and taking real and imaginary parts of $\left(p_{\eta}-i p_{0}\right) Z_{k}$, one can find near the pole

$$
\begin{aligned}
& \left(p_{\eta}+\operatorname{Im} p_{0}\right) \operatorname{Re} Z_{k}+\operatorname{Re} p_{0} \operatorname{Im} Z_{k}=\operatorname{Re} \xi \approx \text { const }, \\
& \operatorname{Re} p_{0} \operatorname{Re} Z_{k}-\left(p_{\eta}+\operatorname{Im} p_{0}\right) \operatorname{Im} Z_{k}=-\operatorname{Im} \xi \approx \text { const }
\end{aligned}
$$

here $k=1, \ldots, 4$ and const means independence of $p_{\eta}$. The magnitudes of $I m p_{0}$ and $R e p_{0}$ are restricted by the quasibound state energy $Q_{0}=-p_{0}^{2} / 2 \mu$, determined from the existing unpolarized data [10]:

$$
2 \mu \operatorname{Re} Q_{0}=\left(\operatorname{Imp}_{0}\right)^{2}-\left(\operatorname{Re} p_{0}\right)^{2}, \mu\left|\operatorname{Im} Q_{0}\right|=\left|\operatorname{Re} p_{0}\right|\left|\operatorname{Im} p_{0}\right|,
$$

where $\mu$ is the reduced mass of the ${ }^{3} \mathrm{He}-\eta$ system. Eqs. (34), (35) and Eq. (36) can be used for more precise determination of the magnitudes of $I m p_{0}$ and $R e p_{0}$ and can give their signs. Knowledge of the sign of the real part of $p_{0}$ allows one to conclude whether the ${ }^{3} \mathrm{He}-\eta$ state is quasi-bound $\left(\operatorname{Re}_{0}>0\right)$ or anti-bound $\left(\operatorname{Re} p_{0}<0\right)$. One should note that at the pole $p_{\eta}=i p_{0}$ defined by Eq. (1), the radial part of the s-wave function of the ${ }^{3} \mathrm{He}-\eta$ relative motion has the asymptotic form of $\sim \exp \left(i p_{\eta} r\right) / r=$ $\exp \left[\left(-\operatorname{Re} p_{0}-i \operatorname{Im} p_{0}\right) r\right] / r$. This function vanishes at $r \rightarrow$ $\infty$ (bound) for $\operatorname{Rep}_{0}>0$ and increases infinitely (antibound) for $R e p_{0}<0$. Note that our definition of $p_{0}$ in Eq. (1) differs from [12] by the imaginary unit $i$.

Measurement of spin transfer coefficients is a complicated experimental problem. In this connection one should note that measurement of only two observables $A_{y}^{d}$ and $A_{x z}$ completely determines the following complex number

$$
\begin{aligned}
Z_{5}= & M_{1} M_{5}^{*}+M_{2} M_{6}^{*}= \\
& =\left[C A^{*}-B D^{*}+\left(|C|^{2}-|D|^{2}\right) q_{z}\right] q_{x} .
\end{aligned}
$$

This can be seen from the relations $\operatorname{Re} Z_{5}=-\frac{I}{3} A_{x z}$ and $\operatorname{Im} Z_{5}=-\frac{I}{2} A_{y}^{d}$, following from Eqs.(13) and (14), respectively. We emphasize that Eq. (37) is valid not only for the case of four spin amplitudes, but also in the general case of six independent amplitudes. However, in the pole region, $Z_{5}$ is a linear combination of the terms $\left(p_{\eta}-i p_{0}\right)^{-1}$ and $\left(p_{\eta}+i p_{0}^{*}\right)^{-1}$

$$
Z_{5}=\frac{\xi_{1}}{p_{\eta}-i p_{0}}+\frac{\xi_{2}}{p_{\eta}+i p_{0}^{*}},
$$

where $\xi_{1}$ and $\xi_{2}$ are unknown smooth functions of $p_{\eta}$. Therefore, Eqs. (34) and (35) are not valid for $Z_{5}$, and, consequently, one cannot determine from this observable the sign of $R e p_{0}$. Nevertheless, this observable can be used as a new crucial test for the existing models of the $p d \rightarrow{ }^{3} \mathrm{He} \eta$ and ${ }^{3} \mathrm{He} \eta \rightarrow{ }^{3} \mathrm{He} \eta$ reactions. As follows from the $t_{20}$ measurement for the reaction $p d \rightarrow{ }^{3} \mathrm{He} \eta[1],|A| \approx|B|$. One can see from Eq. (37), that studying the $q_{z}$ dependence of $Z_{5}$ could allow one to check whether $|C| \approx|D|$. At $q_{z}=0$, $Z_{5}$ gives a certain relation between the s-p phases $\phi_{C A}$ and $\phi_{B D}$.

Finally, there are other two complex numbers, each of them can be determined by measurement of two spincorrelation parameters $C_{i, j}$ and $C_{i, j k}$ :

$$
\begin{aligned}
Z_{6} & =M_{1} M_{3}^{*}-i M_{2} M_{4}^{*}, \\
\operatorname{Re} Z_{6} & =-\frac{I}{3} C_{x, x y}, I m Z_{6}=\frac{I}{2} C_{x, z}, \\
Z_{7} & =M_{4} M_{5}^{*}-i M_{3} M_{6}^{*}, \\
R e Z_{7} & =-\frac{I}{3} C_{z, y z}, I m Z_{7}=\frac{I}{2} C_{z, x} .
\end{aligned}
$$


The variables $Z_{6}$ and $Z_{7}$ are also combinations of the ( $p_{\eta}-$ $\left.i p_{0}\right)^{-1}$ and $\left(p_{\eta}+i p_{0}^{*}\right)^{-1}$ terms like Eq. (38). Note that all variables $Z_{i}(i=1, \ldots, 7)$ vanish in the collinear regime $\left(q_{x}=0\right)$.

\section{Conclusion}

Assuming that s-wave transistion amplitudes of the reaction $p d \rightarrow{ }^{3} \mathrm{He} \eta$ contain the ${ }^{3} \mathrm{He}-\eta$ pole, whereas the $\mathrm{p}-$ wave amplitudes (and other amplitudes with higher orbital momenta) have non-pole dependence, one should expect that knowledge of energy dependence of the s-wave amplitudes near the threshold could allow one to determine the signs of the real and imaginary parts of the pole point in the complex momentum plane. In its turn, this allows one to determine whether the ${ }^{3} \mathrm{He}-\eta$ state is quasi-bound or anti-bound.

In order to determine the s-wave amplitudes, including their moduli and phases, one has to perform either a complete polarization experiment or to find several optimal (specific) observables. We considered both these options. We show that the complete polarization experiment in collinear kinematics suggests only four measurements. However, one cannot separate s- and p-wave amplitudes in collinear regime. Two different cases are considered beyond collinear kinematics. (i) In the case of four spin amplitudes, ten accurate measurements (including spintransfer coefficients) are required to complete the experiment. Knowledge of only analyzing powers and spincorrelation coefficients in the initial state does not allow one to determine moduli of the amplitudes independently of their phases and suggests performing 14 accurate measurements to get all four amplitudes. (ii) In the case of five independent amplitudes, complete polarization experiment suggests 13 accurate measurements. In particular, five moduli of the amplitudes can be determined by measurement of the following five observables $d \sigma_{0}, A_{y y}, A_{x x}$, $K_{x}^{x^{\prime}}(p)$ and $K_{z}^{z^{\prime}}(p)$. Furthermore, for the general case of six spin amplitudes the complete polarization experiment is too cumbersome and practically unrealizable.

In view of this complexity, we suggest to measure those spin observables which allow one to single out the pole dependence of the s-wave amplitudes. There are two sets of such observables: $C_{z, x}, K_{x}^{z^{\prime}}(d), C_{z, y z}, K_{y z}^{z^{\prime}}$ and $C_{x, z}, K_{z}^{x^{\prime}}(d)$, $C_{x, x y}, K_{x y}^{x^{\prime}}$.

In the most realistic case, the analyzing powers $A_{y}^{d}$ and $A_{x z}$ can be measured. It allows one to determine the specific observable which has a two-pole form of Eq. (38) near the threshold and can be used as a crucial test for existing theoretical models of the reaction amplitude.

Finally, one should note that in general case of six independent spin amplitudes in the transtion matrix element given by Eq. (2) the developed formalism is valid for the reactions $p d \rightarrow{ }^{3} \mathrm{H} \pi^{+}$and $p d \rightarrow{ }^{3} \mathrm{He} \pi^{0}$ too.

\section{Acknowledgements}

I am thankful to J. Haidenbauer, A.E. Kudryavtsev and C. Wilkin for useful discussions.

\section{References}

1. J.Berger et al., Phys. Rev. Lett. 61 (1988) 919.

2. B. Mayer et al. Phys. Rev. C 53 (1996) 2068.

3. C. Wilkin, Phys. Rev. C 47 (1993) R938.

4. L.A. Kondratyuk, A.V. Lado, Yu.N. Uzikov, Yad. Fiz. 58 (1995) 524 [Phys. Atom. Nucl. 58 (1995) 473].

5. L.A. Kondratyuk, A.V. Lado, Yu.N. Uzikov, Proc. of the Int. Conf. on Mesons and Nuclei at Intermediate Energies (3-8 May, 1994, Dubna, Russia), Eds. M.Kh. Khankhasaev, Zh.B. Kurmanov, World Scientific, 1995 , p.714.

6. G. Fäldt, C. Wilkin, Nucl. Phys. A 587(1995) 769.

7. S.A. Rakityanskiy, et al. Phys. Rev. C53 (1996) R2043.

8. A. Fix, H. Arenhövel, Phys. Rev. 66 (2002) 024002.

9. V. Baru et. al., Phys. Rev. C 68 (2003) 035203.

10. T. Mersmann et al. Phys. Rev. Lett. 98 (2007) 242301.

11. J. Smyrski et al. Phys. Lett. 649 (2007) 258.

12. C. Wilkin et al., Phys. Lett. B 654 (2007) 92.

13. Yu.N. Uzikov, Nucl.Phys. A 801 (2008) 114.

14. G. Ohlsen, Rep. Prog. Phys. 35 (1972) 717. 